\title{
Yield and Economics of Maize (Zea mays) Preceded by Greengram as Influenced by Irrigation Schedules and Nitrogen Levels
}

\author{
Y. Deepthi Kiran*, V. Sumathi and G. Prabhakara Reddy \\ Department of Agronomy, S. V. Agricultural College, Tirupati-517502, \\ Andhra Pradesh, India \\ *Corresponding author
}

\section{A B S T R A C T}

Keywords

Yield, Gross returns, Net returns, Returns per rupee invested

Article Info

Accepted:

07 October 2019

Available Online:

10 November 2019
A field study was conducted over two years (during kharif and rabi seasons of 2013 and 2014) to evaluate the yield and returns from maize under different irrigation schedules and nitrogen levels at S.V. Agricultural College, wet land farm, Tirupati, Andhra Pradesh. During kharif, greengram was raised as bulk crop. The rabi maize experiment was laid out in a split plot design with three replications by taking four irrigation schedules as main plots and three nitrogen levels as sub plots. Yield of maize was found to be higher with weekly check basin method, which was on par to those obtained with drip irrigation at $0.9 \mathrm{IW} / \mathrm{CPE}$ ratio. With regard to nitrogen levels tried, these values were found to be superior with the nitrogen dose of $240 \mathrm{~kg} \mathrm{~N}^{-1}$. The interaction between the irrigation schedules and nitrogen levels indicated that higher yield was observed with scheduling irrigation either by weekly check basin method or by drip irrigation at 0.9 IW/CPE ratio along with $240 \mathrm{~kg} \mathrm{~N} \mathrm{ha}^{-1}$. But the gross and net returns were higher with drip irrigation scheduled at 0.9 IW/CPE ratio along with $240 \mathrm{~kg} \mathrm{~N} \mathrm{ha}^{-1}$. The experimental results revealed that growing of greengram preceded by maize was economical than maize alone and maize crop can be grown economically with limited water supply at 0.9 IW/CPE ratio through drip irrigation along with $240 \mathrm{~kg} \mathrm{~N} \mathrm{ha}^{-1}$.

\section{Introduction}

Maize, a miracle crop, is grown throughout the year over a wide range of soils in India. Because of its high grain productivity, it has an enormous potentiality in ensuring food security. With reference to Southern Agro Climatic Zone of Andhra Pradesh, besides as a food crop, maize has been gaining importance due to well established market of poultry feed units. Water and nitrogen are two important resources for maize production. Yields in maize respond positively with an increase in the amount of water and nitrogen applied and reaches the plateau at their optimum doses. It has been reported, that maize grown under limited water supply requires less nitrogen to achieve maximum grain yield than that required 
with well water supply (Moser et al., 2006). Meager information is available on scheduling of irrigation through drip and nitrogen rates in maize. Hence, the present study was under taken to examine the appropriate irrigation schedule through drip as well as nitrogen application rate.

\section{Materials and Methods}

A field experiment was carried out during kharif and rabi seasons of 2013-14 and 201415 at S.V. Agricultural College, wet land farm, Tirupati. The experimental site was located at $13.5^{\circ} \mathrm{N}$ latitude, $79.3^{\circ} \mathrm{E}$ longitude and at an altitude of $182.9 \mathrm{~m}$ above the mean sea level, in the Southern Agro-climatic Zone of Andhra Pradesh. The soil was sandy loam in texture, neutral in reaction (7.9), low in organic carbon $(0.25 \%)$ and available nitrogen $\left(178 \mathrm{~kg} \mathrm{ha}{ }^{-1}\right)$ and medium in available phosphorus (24.9 $\left.\mathrm{kg} \mathrm{ha}^{-1}\right)$ and available potassium (174 $\mathrm{kg} \mathrm{ha}^{-1}$ ). During kharif, greengram was raised as bulk crop by following the recommended package of practices. After manual picking of greengram pods, the haulms were incorporated in to the soil. The rabi maize experiment was laid out in a split plot design with three replications. The treatments comprised of four main plots viz., $\mathrm{M}_{1}$ (drip irrigation at $0.7 \mathrm{IW} / \mathrm{CPE}$ ratio), $\mathrm{M}_{2}$ (drip irrigation at $0.8 \mathrm{IW} / \mathrm{CPE}$ ratio), $\mathrm{M}_{3}$ (drip irrigation at $0.9 \mathrm{IW} / \mathrm{CPE}$ ratio) and $\mathrm{M}_{4}$ (weekly check basin irrigation) and three sub plots viz., $\mathrm{N}_{1}\left(160 \mathrm{~kg} \mathrm{~N} \mathrm{ha}^{-1}\right), \mathrm{N}_{2}(200 \mathrm{~kg} \mathrm{~N}$ $\mathrm{ha}^{-1}$ ) and $\mathrm{N}_{3}$ (240 $\left.\mathrm{kg} \mathrm{N} \mathrm{ha}{ }^{-1}\right)$. Separately, unreplicated observational plot was also maintained with same treatments to maize but without preceding greengram for comparative study. The test variety of maize was DHM117. Paired rows were made by hand hoe with a distance of $60 / 30 \mathrm{~cm}$ and seeds were dibbled @ 2 seeds hill $^{-1}$ at a spacing of $20 \mathrm{~cm}$. Nitrogen was applied as per the prescribed sub plot treatments in three splits i.e., half at basal, one fourth at knee high stage and remaining one fourth at tasseling stage. Recommended dose of phosphorus and potassium each @80 $\mathrm{kg} \mathrm{ha}^{-1}$ were applied basally. Atrazine @ 1.25 $\mathrm{kg}$ a.i ha ${ }^{-1}$ was sprayed as pre-emergence to control the weed emergence during early stages of crop growth. One hand weeding was done during vegetative stage to control the weeds at later stages. All other recommended package of practices for growing of maize was followed. Irrigations were scheduled as per the prescribed sub plot treatments. To schedule the drip irrigation at prescribed IW/CPE ratios the treatments were maintained to field capacity in the top $0-45 \mathrm{~cm}$ depth i.e. only effective root zone depth, whereas for check basin irrigation the depth of water was $50 \mathrm{~cm}$. The quantity of water applied to each plot was determined by considering number of laterals, emitter spacing and discharge rate. The total quantity of water applied at different IW/CPE ratios was kept the same (Table 1).

Total quantity of water applied (litres)= $\frac{\text { Plot size }\left(\mathrm{m}^{2}\right) \times \text { depth of water }(\mathrm{mm})}{1000} \times 1000$

\section{Results and Discussion}

\section{Yield}

Yields (kernel and straw) of maize were significantly influenced by the irrigation schedules and nitrogen levels as well as their interaction, during both the years of investigation. The highest yields (grain and straw) of maize were recorded under weekly check basin irrigation $\left(\mathrm{M}_{4}\right)$, which was on par with those recorded under IW/CPE ratio of 0.9 through drip irrigation $\left(\mathrm{M}_{3}\right)$ than rest of the irrigation schedules tried. In weekly check basin irrigation, because of adequate turgidity inside the plant, the growth and development of cob was better. In addition to this, adequate turgidity prevailed inside the plant might have been congenial to translocate the photosynthates efficiently from the source to 
the development of sink consequently yield (Paolo and Rinaldi 2008). Increase in kernel yield under drip irrigation at 0.9 IW/CPE was mainly due to increased soil moisture in the upper $30 \mathrm{~cm}$ soil layer, leading to higher plant relative water content and less negative leaf water potential as quoted by Viswanatha et al., (2002). The decrease in tissue water potential affects the several physiological processes. Plant water deficit affects the final yield through its influence on various physiological processes. These results are in conformity with the findings of Aulakh et al., (2013). Among the nitrogen levels, application of nitrogen at $240 \mathrm{~kg} \mathrm{ha}\left(\mathrm{N}_{3}\right)$ produced significantly higher values of all the yield attributes and yields as compared to the lower doses of nitrogen. This might be because of better pollination under higher nitrogen levels, helping to maintain the sink capacity thereby causing well filled kernels in cob (Zakkam et al., 2012) (Table 2).

Regarding interaction, weekly irrigations with $240 \mathrm{~kg} \mathrm{~N}$ ha $^{-1}\left(\mathrm{M}_{4} \mathrm{~N}_{3}\right)$ registered the highest yield (grain and straw), which were statistically on par to those recorded with the combination of drip irrigation at $0.9 \mathrm{IW} / \mathrm{CPE}$ ratio and $240 \mathrm{~kg} \mathrm{~N}^{-1}\left(\mathrm{M}_{3} \mathrm{~N}_{3}\right)$, during both the years of study. This might be due to the combined effect of adequate moisture under high nitrogen level, leading to better partitioning and translocation of photosynthates from source to sink (Singh, 2001). Drip irrigation at $0.7 \mathrm{IW} / \mathrm{CPE}$ ratio with nitrogen level of $160 \mathrm{~kg} \mathrm{ha}^{-1}\left(\mathrm{M}_{1} \mathrm{~N}_{1}\right)$ recorded the lowest stature of yield components and yield during both the years.

\section{Economics}

Influence of different irrigation schedules and nitrogen levels on economics of maize as well as greengram-maize cropping system in terms of gross returns and net returns were worked out and presented below. The trend in economics of maize crop alone was similar to that of economics realized with the greengram-maize cropping system, during both the years of study.

Gross returns and net returns were realized under maize preceded by greengram with crop residue incorporation were relatively low as compared to no residue incorporation.

\section{Gross returns}

Different irrigation schedules and nitrogen levels as well as their interaction exerted significant influence on gross returns, during both the years of study, with unaltered trend (Table 3 and 4). The higher gross returns were realized with weekly check basin irrigation, which was on par to that obtained with irrigation at IW/CPE ratio of 0.9 through drip and both of them were significantly superior to drip irrigation at $0.8 \mathrm{IW} / \mathrm{CPE}$ ratio. The lowest gross returns were realized with drip irrigation at $0.7 \mathrm{IW} / \mathrm{CPE}$ ratio. Among the nitrogen levels, application of higher level of nitrogen at $240 \mathrm{~kg} \mathrm{ha}^{-1}$ accrued significantly higher gross returns than the remaining doses. The next best treatment was application of nitrogen at $200 \mathrm{~kg} \mathrm{ha}^{-1}$, whereas the lower gross returns were realized with nitrogen level of 160 $\mathrm{kg} \mathrm{ha}^{-1}$.

Interaction indicated that higher levels of irrigation schedules produced on par gross returns at any level of nitrogen. Higher nitrogen doses of 240 and $200 \mathrm{~kg} \mathrm{ha}^{-1}$ realized the comparable gross returns at any irrigation level, during both the years. A treatment combination of weekly check basin irrigation and $240 \mathrm{~kg} \mathrm{~N} \mathrm{ha}{ }^{-1}$ produced higher gross returns, which was statistically on par with the treatment combination of drip irrigation at IW/CPE ratio of 0.8 along with the same dose of nitrogen and with weekly check basin irrigation along with $200 \mathrm{~kg} \mathrm{ha}^{-1}$. The lowest gross returns were resulted with the 
combination of drip irrigation at $0.7 \mathrm{IW} / \mathrm{CPE}$ ratio along with $160 \mathrm{~kg} \mathrm{~N} \mathrm{ha}^{-1}$.

\section{Net returns}

Irrigation schedules, levels of nitrogen and their interaction exerted significant effect on net returns from maize alone and greengrammaize cropping system as well following similar trend, during both the years of study (Table 5 and 6). Net returns were found to be the highest with the scheduling of irrigation through drip irrigation at 0.9 IW/CPE ratio followed by weekly irrigation, with no significant difference between them and both of them were significantly superior to drip irrigation at IW/CPE ratio of 0.8 , during both the years. Significantly, the lowest net returns were recorded with drip irrigation at IW/CPE ratio of 0.7.Application of $240 \mathrm{~kg} \mathrm{ha}^{-1}$ resulted in higher net returns, which was significantly superior to $200 \mathrm{~kg} \mathrm{ha}^{-1}$, while the lowest net returns were noticed with $160 \mathrm{~kg} \mathrm{~N} \mathrm{ha}^{-1}$.

Regarding the interaction effect, net returns were higher with frequent irrigation regimes along with the nitrogen doses of 200 and 240 $\mathrm{kg} \mathrm{ha}^{-1}$. The higher net returns were obtained with drip irrigation at $0.9 \mathrm{IW} / \mathrm{CPE}$ ratio with nitrogen dose of $240 \mathrm{~kg} \mathrm{ha}^{-1}$, which was comparable with weekly check basin method of irrigation at same nitrogen dose and drip irrigation at $0.9 \mathrm{IW} / \mathrm{CPE}$ with $200 \mathrm{~kg} \mathrm{~N} \mathrm{ha}^{-1}$.
However, the lowest net returns were observed under drip irrigation at IW/CPE of 0.7 along with $160 \mathrm{~kg} \mathrm{~N} \mathrm{ha}^{-1}$, during both the years of study.

Among the irrigation schedules, the higher gross returns realized with weekly irrigations, which was on par to that of drip irrigation at IW/CPE ratio of 0.9. This might be due to increased yields under higher irrigation levels. The net returns were higher with drip irrigation at $0.9 \mathrm{IW} / \mathrm{CPE}$ ratio, which was statistically on par with the weekly irrigations during both the years of study. Drip irrigation at $0.7 \mathrm{IW} / \mathrm{CPE}$ ratio resulted in the lowest monetary returns. Relatively higher moisture content increased the grain and stover yield, which finally reflected in the gross returns and net returns. On the other hand, where the crop was subjected to moisture stress, ultimately resulted in lower monetary returns. Similar findings have been reported by Reddy et al., (2012) and Reddy and Padmaja (2014).

With regard to nitrogen levels, increase in nitrogen from 160 to $240 \mathrm{~kg} \mathrm{ha}^{-1}$ significantly increased the gross returns and net returns. This might be because of increased yields under higher nitrogen levels which might have resulted in the remunerative returns. These results corroborate with the findings of Reddy et al., (2012), Paramasivan et al., (2013) and Om et al., (2014).

Table.1 The details of drip irrigation is furnished below

\begin{tabular}{|c|c|c|c|c|c|}
\hline Treatments & $\begin{array}{c}\text { Cumulative Epan } \\
\text { for irrigation } \\
(\mathbf{m m})\end{array}$ & $\begin{array}{c}\text { Lateral } \\
\text { spacing } \\
(\mathbf{c m})\end{array}$ & $\begin{array}{c}\text { Emitter } \\
\text { spacing } \\
(\mathbf{c m})\end{array}$ & $\begin{array}{c}\text { Flow } \\
\text { rate } \\
\left(\mathbf{l ~ h r} \mathbf{~}^{\mathbf{- 1}}\right)\end{array}$ & $\begin{array}{c}\text { Irrigation } \\
\text { duration } \\
\text { (hours) }\end{array}$ \\
\hline IW/CPE of $\mathbf{0 . 7}$ & 50.0 & 90 & 40 & 4 & 3.24 \\
\hline IW/CPE of $\mathbf{0 . 8}$ & 43.7 & 90 & 40 & 4 & 3.24 \\
\hline IW/CPE of $\mathbf{0 . 9}$ & 38.8 & 90 & 40 & 4 & 3.24 \\
\hline
\end{tabular}


Table.2 Kernel yield $\left(\mathrm{kg} \mathrm{ha}^{-1}\right)$ of maize as influenced by irrigation schedules and nitrogen levels under drip irrigation preceded by greengram

\begin{tabular}{|c|c|c|c|c|c|c|c|c|}
\hline \multirow[t]{2}{*}{ Treatments } & \multicolumn{4}{|c|}{ 2013-14 } & \multicolumn{4}{|c|}{ 2014-15 } \\
\hline & $\mathrm{N}_{1}: 160$ & $\mathrm{~N}_{2}: 200$ & $\mathrm{~N}_{3}: 240$ & Mean & $\mathrm{N}_{1}: 160$ & $\mathrm{~N}_{2}: 200$ & $\mathrm{~N}_{3}: 240$ & Mean \\
\hline $\mathrm{M}_{1}: 0.7 \mathrm{IW} / \mathrm{CPE}$ & 2073 & 2743 & 2929 & 2582 & 2339 & 2763 & 3049 & 2717 \\
\hline $\mathrm{M}_{2}: 0.8 \mathrm{IW} / \mathrm{CPE}$ & 3063 & 4065 & 4194 & 3774 & 3101 & 4103 & 4236 & 3813 \\
\hline $\mathrm{M}_{3}: 0.9 \mathrm{IW} / \mathrm{CPE}$ & 3759 & 4907 & 5051 & 4572 & 3723 & 4838 & 5015 & 4525 \\
\hline$M_{4}:$ Weekly check basin & 3813 & 4948 & 5128 & 4630 & 3992 & 4976 & 5204 & 4724 \\
\hline \multirow[t]{2}{*}{ Mean } & 3177 & 4166 & 4326 & & 3289 & 4170 & 4376 & \\
\hline & \multicolumn{2}{|c|}{ S.Em \pm} & \multicolumn{2}{|c|}{$\mathrm{CD}(\mathrm{P}=\mathbf{0 . 0 5})$} & \multicolumn{2}{|c|}{ S.Em \pm} & \multicolumn{2}{|c|}{$\mathrm{CD}(\mathrm{P}=0.05)$} \\
\hline $\mathbf{M}$ & \multicolumn{2}{|c|}{128} & \multicolumn{2}{|c|}{453} & \multicolumn{2}{|c|}{141} & \multicolumn{2}{|c|}{498} \\
\hline $\mathbf{N}$ & \multicolumn{2}{|c|}{34} & \multicolumn{2}{|c|}{102} & \multicolumn{2}{|c|}{40} & \multicolumn{2}{|c|}{122} \\
\hline $\mathbf{M}$ at $\mathbf{N}$ & \multicolumn{2}{|c|}{140} & \multicolumn{2}{|c|}{482} & \multicolumn{2}{|c|}{155} & \multicolumn{2}{|c|}{536} \\
\hline $\mathbf{N}$ at $\mathbf{M}$ & \multicolumn{2}{|c|}{223} & \multicolumn{2}{|c|}{472} & \multicolumn{2}{|c|}{244} & \multicolumn{2}{|c|}{517} \\
\hline \multicolumn{9}{|c|}{ Kernel yield $\left(\mathrm{kg} \mathrm{ha}^{-1}\right)$ of maize in unreplicated observational plot without greengram } \\
\hline $\mathrm{M}_{1}: 0.7 \mathrm{IW} / \mathrm{CPE}$ & 1986 & 2578 & 2636 & 2400 & 2128 & 2652 & 2745 & 2508 \\
\hline$M_{2}: 0.8$ IW/CPE & 2834 & 3742 & 3979 & 3518 & 2984 & 3827 & 4015 & 3609 \\
\hline $\mathrm{M}_{3}: 0.9 \mathrm{IW} / \mathrm{CPE}$ & 3658 & 4769 & 4978 & 4468 & 3694 & 4786 & 4985 & 4488 \\
\hline$M_{4}:$ Weekly check basin & 3774 & 4815 & 5082 & 4557 & 3754 & 4886 & 5116 & 4585 \\
\hline Mean & 3063 & 3976 & 4169 & & 3140 & 4038 & 4108 & \\
\hline
\end{tabular}


Table.3 Stover yield $\left(\mathrm{kg} \mathrm{ha}^{-1}\right)$ of maize as influenced by irrigation schedules and nitrogen levels under drip irrigation preceded by greengram

\begin{tabular}{|c|c|c|c|c|c|c|c|c|}
\hline \multirow[t]{2}{*}{ Treatments } & \multicolumn{4}{|c|}{ 2013-14 } & \multicolumn{4}{|c|}{ 2014-15 } \\
\hline & $\mathrm{N}_{1}: 160$ & $N_{2}: 200$ & $\mathrm{~N}_{3}: \mathbf{2 4 0}$ & Mean & $\mathrm{N}_{1}: 160$ & $\mathrm{~N}_{2}: 200$ & $\mathrm{~N}_{3}: 240$ & Mean \\
\hline $\mathrm{M}_{1}: 0.7 \mathrm{IW} / \mathrm{CPE}$ & 2458 & 3635 & 4078 & 3390 & 2897 & 3714 & 4298 & 3636 \\
\hline $\mathrm{M}_{2}: 0.8 \mathrm{IW} / \mathrm{CPE}$ & 3920 & 5812 & 6333 & 5355 & 4035 & 5852 & 7026 & 5637 \\
\hline$M_{3}: 0.9$ IW/CPE & 5059 & 7420 & 8095 & 6858 & 5094 & 7301 & 8079 & 6825 \\
\hline$M_{4}:$ Weekly check basin & 5383 & 7945 & 8700 & 7343 & 5702 & 7929 & 8911 & 7514 \\
\hline \multirow{2}{*}{ Mean } & 4205 & 6203 & 6801 & & 4432 & 6199 & 7078 & \\
\hline & \multicolumn{2}{|c|}{ S.Em \pm} & \multicolumn{2}{|c|}{$\mathrm{CD}(\mathrm{P}=0.05)$} & \multicolumn{2}{|c|}{ S.Em \pm} & \multicolumn{2}{|c|}{$\mathrm{CD}(\mathrm{P}=\mathbf{0 . 0 5})$} \\
\hline $\mathbf{M}$ & \multicolumn{2}{|c|}{190} & \multicolumn{2}{|c|}{671} & \multicolumn{2}{|c|}{222} & \multicolumn{2}{|c|}{784} \\
\hline $\mathbf{N}$ & \multicolumn{2}{|c|}{52} & \multicolumn{2}{|c|}{158} & \multicolumn{2}{|c|}{120} & \multicolumn{2}{|c|}{364} \\
\hline $\mathbf{M}$ at $\mathbf{N}$ & \multicolumn{2}{|c|}{208} & \multicolumn{2}{|c|}{718} & \multicolumn{2}{|c|}{297} & \multicolumn{2}{|c|}{982} \\
\hline $\mathbf{N}$ at $\mathbf{M}$ & \multicolumn{2}{|c|}{329} & \multicolumn{2}{|c|}{697} & \multicolumn{2}{|c|}{385} & \multicolumn{2}{|c|}{797} \\
\hline \multicolumn{9}{|c|}{ Stover yield $\left(\mathrm{kg} \mathrm{ha}^{-1}\right)$ of maize unreplicated observational plot without greengram } \\
\hline $\mathrm{M}_{1}: 0.7 \mathrm{IW} / \mathrm{CPE}$ & 2681 & 3558 & 3664 & 3301 & 2875 & 3670 & 3820 & 3455 \\
\hline $\mathrm{M}_{2}: 0.8 \mathrm{IW} / \mathrm{CPE}$ & 3883 & 5238 & 5650 & 4924 & 4091 & 5457 & 5712 & 5087 \\
\hline $\mathrm{M}_{3}: 0.9 \mathrm{IW} / \mathrm{CPE}$ & 5231 & 7153 & 7716 & 6700 & 5316 & 7195 & 7750 & 6754 \\
\hline$M_{4}:$ Weekly check basin & 5925 & 7704 & 7878 & 7169 & 5956 & 7845 & 7925 & 7242 \\
\hline Mean & 4430 & 5913 & 6227 & & 4560 & 6042 & 6312 & \\
\hline
\end{tabular}


Table.4 Gross returns $\left(\mathrm{Cha}^{-1}\right.$ ) of maize as influenced by irrigation schedules and nitrogen levels under drip irrigation preceded by greengram

\begin{tabular}{|c|c|c|c|c|c|c|c|c|}
\hline \multirow[t]{2}{*}{ Treatments } & \multicolumn{4}{|c|}{ 2013-14 } & \multicolumn{4}{|c|}{ 2014-15 } \\
\hline & $\mathrm{N}_{1}: \mathbf{1 6 0}$ & $\mathbf{N}_{2}: 200$ & $\mathrm{~N}_{3}: 240$ & Mean & $\mathrm{N}_{1}: \mathbf{1 6 0}$ & $\mathrm{N}_{2}: \mathbf{2 0 0}$ & $\mathrm{N}_{3}: 240$ & Mean \\
\hline $\mathrm{M}_{1}: 0.7 \mathrm{IW} / \mathrm{CPE}$ & 35626 & 47528 & 50953 & 44703 & 40327 & 47927 & 53093 & 47116 \\
\hline $\mathrm{M}_{2}: 0.8 \mathrm{IW} / \mathrm{CPE}$ & 52930 & 70853 & 73446 & 65743 & 53662 & 71506 & 74807 & 66658 \\
\hline $\mathrm{M}_{3}: 0.9 \mathrm{IW} / \mathrm{CPE}$ & 65214 & 85938 & 88911 & 80021 & 64673 & 84715 & 88325 & 79238 \\
\hline$M_{4}:$ Weekly check basin & 66397 & 87124 & 90759 & 81427 & 69575 & 87556 & 92181 & 83104 \\
\hline \multirow[t]{2}{*}{ Mean } & 55042 & 72861 & 76017 & & 57059 & 72926 & 77102 & \\
\hline & \multicolumn{2}{|c|}{ S.Em \pm} & \multicolumn{2}{|c|}{$\mathrm{CD}(\mathrm{P}=\mathbf{0 . 0 5})$} & \multicolumn{2}{|c|}{ S.Em \pm} & \multicolumn{2}{|c|}{$\mathrm{CD}(\mathrm{P}=\mathbf{0 . 0 5})$} \\
\hline $\mathbf{M}$ & \multicolumn{2}{|c|}{2246} & \multicolumn{2}{|c|}{7923} & \multicolumn{2}{|c|}{2455} & \multicolumn{2}{|c|}{8663} \\
\hline $\mathbf{N}$ & \multicolumn{2}{|c|}{596} & \multicolumn{2}{|c|}{1804} & \multicolumn{2}{|c|}{727} & \multicolumn{2}{|c|}{2200} \\
\hline $\mathbf{M}$ at $\mathbf{N}$ & \multicolumn{2}{|c|}{2448} & \multicolumn{2}{|c|}{8441} & \multicolumn{2}{|c|}{2728} & \multicolumn{2}{|c|}{9363} \\
\hline $\mathbf{N}$ at $\mathbf{M}$ & \multicolumn{2}{|c|}{3890} & \multicolumn{2}{|c|}{8246} & \multicolumn{2}{|c|}{4253} & \multicolumn{2}{|c|}{9016} \\
\hline \multicolumn{9}{|c|}{ Gross returns $\left(\mathrm{ha}^{-1}\right)$ of maize in unreplicated observational plot without greengram } \\
\hline $\mathrm{M}_{1}: 0.7 \mathrm{IW} / \mathrm{CPE}$ & 34457 & 44806 & 45840 & 41701 & 36923 & 46102 & 47740 & 43588 \\
\hline $\mathrm{M}_{2}: 0.8 \mathrm{IW} / \mathrm{CPE}$ & 49227 & 65110 & 69314 & 61217 & 51835 & 66689 & 69952 & 62825 \\
\hline $\mathrm{M}_{3}: 0.9 \mathrm{IW} / \mathrm{CPE}$ & 63759 & 83457 & 87364 & 78193 & 64420 & 83771 & 87510 & 78567 \\
\hline$M_{4}:$ Weekly check basin & 66309 & 84744 & 89190 & 80081 & 66020 & 86021 & 89781 & 80607 \\
\hline Mean & 53438 & 69529 & 72927 & & 54800 & 70646 & 73746 & \\
\hline
\end{tabular}


Table.5 Gross returns ( $₹ \mathrm{ha}^{-1}$ ) of greengram-maize cropping system as influenced by irrigation schedules and nitrogen levels under drip irrigation

\begin{tabular}{|c|c|c|c|c|c|c|c|c|}
\hline \multirow{2}{*}{ Treatments } & \multicolumn{4}{|c|}{ 2013-14 } & \multicolumn{4}{|c|}{ 2014-15 } \\
\hline & $\mathrm{N}_{1}: \mathbf{1 6 0}$ & $\mathbf{N}_{2}: 200$ & $\mathrm{~N}_{3}: 240$ & Mean & $\mathrm{N}_{1}: 160$ & $\mathrm{~N}_{2}: 200$ & $\mathrm{~N}_{3}: 240$ & Mean \\
\hline $\mathrm{M}_{1}: 0.7 \mathrm{IW} / \mathrm{CPE}$ & 59916 & 71818 & 75243 & 68993 & 65387 & 72987 & 78153 & 72176 \\
\hline $\mathrm{M}_{2}: 0.8 \mathrm{IW} / \mathrm{CPE}$ & 77220 & 95143 & 97736 & 90033 & 78722 & 96566 & 99867 & 91718 \\
\hline $\mathrm{M}_{3}: 0.9 \mathrm{IW} / \mathrm{CPE}$ & 89504 & 110228 & 113201 & 104311 & 89733 & 109775 & 113385 & 104298 \\
\hline$M_{4}:$ Weekly check basin & 90687 & 111414 & 115049 & 105717 & 94635 & 112616 & 117241 & 108164 \\
\hline \multirow[t]{2}{*}{ Mean } & 79332 & 97151 & 100307 & & 82119 & 97986 & 102162 & \\
\hline & \multicolumn{2}{|c|}{ S.Em \pm} & \multicolumn{2}{|c|}{$\mathrm{CD}(\mathrm{P}=\mathbf{0 . 0 5})$} & \multicolumn{2}{|c|}{ S.Em \pm} & \multicolumn{2}{|c|}{$\mathrm{CD}(\mathrm{P}=\mathbf{0 . 0 5})$} \\
\hline M & \multicolumn{2}{|c|}{2246} & \multicolumn{2}{|c|}{7923} & \multicolumn{2}{|c|}{2455} & \multicolumn{2}{|c|}{8663} \\
\hline $\mathbf{N}$ & \multicolumn{2}{|c|}{596} & \multicolumn{2}{|c|}{1804} & \multicolumn{2}{|c|}{727} & \multicolumn{2}{|c|}{2200} \\
\hline $\mathbf{M}$ at $\mathbf{N}$ & \multicolumn{2}{|c|}{2448} & \multicolumn{2}{|c|}{8441} & \multicolumn{2}{|c|}{2728} & \multicolumn{2}{|c|}{9363} \\
\hline $\mathbf{N}$ at $\mathbf{M}$ & \multicolumn{2}{|c|}{3890} & \multicolumn{2}{|c|}{8246} & \multicolumn{2}{|c|}{4253} & \multicolumn{2}{|c|}{9016} \\
\hline
\end{tabular}


Table.6 Net returns (₹ ha $^{-1}$ ) of maize as influenced by irrigation schedules and nitrogen levels under drip irrigation preceded by greengram

\begin{tabular}{|c|c|c|c|c|c|c|c|c|}
\hline \multirow[t]{2}{*}{ Treatments } & \multicolumn{4}{|c|}{ 2013-14 } & \multicolumn{4}{|c|}{ 2014-15 } \\
\hline & $\mathrm{N}_{1}: 160$ & $\mathrm{~N}_{2}: 200$ & $\mathrm{~N}_{3}: 240$ & Mean & $N_{1}: 160$ & $\mathrm{~N}_{2}: \mathbf{2 0 0}$ & $N_{3}: 240$ & Mean \\
\hline $\mathrm{M}_{1}: 0.7 \mathrm{IW} / \mathrm{CPE}$ & 1557 & 12964 & 15894 & 10139 & 6258 & 13363 & 18034 & 12552 \\
\hline $\mathrm{M}_{2}: 0.8 \mathrm{IW} / \mathrm{CPE}$ & 18200 & 35628 & 37726 & 30518 & 18932 & 36281 & 39087 & 31433 \\
\hline $\mathrm{M}_{3}: 0.9 \mathrm{IW} / \mathrm{CPE}$ & 29824 & 50053 & 52531 & 44136 & 29283 & 48830 & 51945 & 43353 \\
\hline$M_{4}:$ Weekly check basin & 27545 & 47777 & 50917 & 42080 & 29721 & 47207 & 51337 & 42755 \\
\hline \multirow[t]{2}{*}{ Mean } & 19282 & 36605 & 39267 & & 21048 & 36420 & 40101 & \\
\hline & \multicolumn{2}{|c|}{ S.Em \pm} & \multicolumn{2}{|c|}{$\mathrm{CD}(\mathrm{P}=\mathbf{0 . 0 5})$} & \multicolumn{2}{|c|}{ S.Em \pm} & \multicolumn{2}{|c|}{$\mathrm{CD}(\mathrm{P}=\mathbf{0 . 0 5})$} \\
\hline $\mathbf{M}$ & \multicolumn{2}{|c|}{2246} & \multicolumn{2}{|c|}{7923} & \multicolumn{2}{|c|}{2455} & \multicolumn{2}{|c|}{8663} \\
\hline $\mathbf{N}$ & \multicolumn{2}{|c|}{596} & \multicolumn{2}{|c|}{1804} & \multicolumn{2}{|c|}{727} & \multicolumn{2}{|c|}{2200} \\
\hline $\mathbf{M}$ at $\mathbf{N}$ & \multicolumn{2}{|c|}{2448} & \multicolumn{2}{|c|}{8441} & \multicolumn{2}{|c|}{2728} & \multicolumn{2}{|c|}{9363} \\
\hline $\mathbf{N}$ at $\mathbf{M}$ & \multicolumn{2}{|c|}{3890} & \multicolumn{2}{|c|}{8246} & \multicolumn{2}{|c|}{4253} & \multicolumn{2}{|c|}{9016} \\
\hline \multicolumn{9}{|c|}{ Net returns $\left(\mathrm{ha}^{-1}\right)$ of maize in unreplicated observational plot without greengram } \\
\hline $\mathrm{M}_{1}: 0.7 \mathrm{IW} / \mathrm{CPE}$ & 388 & 10242 & 10781 & 7137 & 2854 & 11538 & 12681 & 9024 \\
\hline $\mathrm{M}_{2}: 0.8 \mathrm{IW} / \mathrm{CPE}$ & 14497 & 29885 & 33594 & 25992 & 17105 & 31464 & 34232 & 27600 \\
\hline $\mathrm{M}_{3}: 0.9 \mathrm{IW} / \mathrm{CPE}$ & 28369 & 47572 & 50984 & 42308 & 29030 & 47886 & 51130 & 42682 \\
\hline$M_{4}:$ Weekly check basin & 27457 & 45397 & 49348 & 40734 & 26166 & 45672 & 48937 & 40258 \\
\hline Mean & 17678 & 33274 & 36177 & & 18789 & 34140 & 36745 & \\
\hline
\end{tabular}


Table.7 Net returns ( $^{-1} \mathrm{a}^{-1}$ ) of greengram-maize cropping system as influenced by irrigation schedules and nitrogen levels under drip irrigation

\begin{tabular}{|c|c|c|c|c|c|c|c|c|}
\hline \multirow{2}{*}{ Treatments } & \multicolumn{9}{|c|}{$\mathbf{2 0 1 3 - 1 4}$} & \multicolumn{4}{|c|}{$\mathbf{2 0 1 4 - 1 5}$} \\
\hline & $\mathbf{N}_{\mathbf{1}}: \mathbf{1 6 0}$ & $\mathbf{N}_{\mathbf{2}}: \mathbf{2 0 0}$ & $\mathbf{N}_{\mathbf{3}}: \mathbf{2 4 0}$ & Mean & $\mathbf{N}_{\mathbf{1}}: \mathbf{1 6 0}$ & $\mathbf{N}_{\mathbf{2}}: \mathbf{2 0 0}$ & $\mathbf{N}_{\mathbf{3}}: \mathbf{2 4 0}$ & $\mathbf{M e a n}$ \\
\hline $\mathbf{M}_{\mathbf{1}}: \mathbf{0 . 7}$ IW/CPE & 12340 & 23747 & 26677 & 20922 & 17811 & 24916 & 29587 & $\mathbf{2 4 1 0 5}$ \\
\hline $\mathbf{M}_{\mathbf{2}}: \mathbf{0 . 8}$ IW/CPE & 28983 & 46411 & 48509 & 41301 & 30485 & 47834 & 50640 & $\mathbf{4 2 9 8 6}$ \\
\hline $\mathbf{M}_{\mathbf{3}}: \mathbf{0 . 9}$ IW/CPE & 40607 & 60836 & 63314 & 54919 & 40836 & 60383 & 63498 & $\mathbf{5 4 9 0 6}$ \\
\hline $\mathbf{M}_{\mathbf{4}}:$ Weekly check basin & 38328 & 58560 & 61700 & 52863 & 41274 & 58760 & 62890 & $\mathbf{5 4 3 0 8}$ \\
\hline Mean & $\mathbf{3 0 0 6 5}$ & $\mathbf{4 7 3 8 8}$ & $\mathbf{5 0 0 5 0}$ & & $\mathbf{3 2 6 0 1}$ & $\mathbf{4 7 9 7 3}$ & $\mathbf{5 1 6 5 4}$ & \\
\hline
\end{tabular}

\begin{tabular}{|c|c|c|c|c|}
\hline & S.Em \pm & CD $(\mathbf{P}=\mathbf{0 . 0 5})$ & S.Em \pm & $\mathbf{C D}(\mathbf{P}=\mathbf{0 . 0 5})$ \\
\hline $\mathbf{M}$ & 2246 & 7923 & 2455 & $\mathbf{8 6 6 3}$ \\
\hline $\mathbf{N}$ & 596 & 1804 & 727 & $\mathbf{2 2 0 0}$ \\
\hline M at N & 2448 & 8441 & 2728 & $\mathbf{9 3 6 3}$ \\
\hline N at $\mathbf{M}$ & $\mathbf{3 8 9 0}$ & $\mathbf{8 2 4 6}$ & $\mathbf{4 2 5 3}$ & $\mathbf{9 0 1 6}$ \\
\hline
\end{tabular}


The highest monetary returns under higher irrigation regimes of 0.9 IW/CPE ratios through drip and weekly irrigations using check basin method along with higher nitrogen doses might be due to increased kernel and stover yields under favourable moisture conditions coupled with ample supply of nitrogen (Table 7). The lowest monetary returns were noticed in drip irrigation at $0.7 \mathrm{IW} / \mathrm{CPE}$ ratio along with 160 $\mathrm{kg} \mathrm{N} \mathrm{ha}{ }^{-1}$, which might be due to reduced yields. Present investigations confirmed the results of Pennaiah (2005).

Remunerative returns obtained with the greengram-maize cropping system along with crop residue incorporation, were found to be more than with no residue incorporation.

This was due to the reason that additional yield and monetary returns from greengram increased returns from the system. These results are supported by the findings of Devkota et al., (2006), Mala (2008), Sharma and Behera (2009).

Based on the outcome of the investigation, it could be inferred that for maximum yield, and monetary returns drip irrigation scheduled at 0.9 IW/CPE ratio coupled with $240 \mathrm{~kg} \mathrm{~N}^{-1}$ was the better combination under limited water conditions. Growing greengram, as a preceding crop and incorporation of residues after realizing the economic produce will be a promising option for maintaining soil fertility status with additional advantage of greengram seed.

\section{References}

Aulakh, G.S., Vashist, K.K and Mahal, S.S. 2013. Effect of different irrigation regimes and nitrogen levels on growth parameters and yield of late kharif sown maize (Zea mays L.). Crop Research. 45(1, 2 \& 3): 96-105.
Devkota, K.P., Yadav, D.N., Chaudhary, N.K., Dangol, D.R and Basnet, K. B. 2006. Influence of spring season crop residue on productivity of rice-wheat cropping system. Journal of International Agriculture and Animal Science. 27: 53-58.

Mala, S. 2008. Response of hybrid maize to crop residue incorporation in combination with graded levels of nitrogen. Ph.D Thesis submitted to Acharya N.G. Ranga Agricultural University, Rajendranagar, Hyderabad, Andhra Pradesh.

Moser, S.B., Feil, B., Jampatong, S. and Stamp, P. 2006. Effects of pre-anthesis drought, nitrogen fertilizer rate and variety on grain yield, yield components and harvest index of tropical maize. Agricultural Water Management.81: 41-58.

Om, H., Singh, S.P., Singh, J.K., Singh, R.N., Ansari, M.A., Meena, R.L and Yadav, B. 2014. Productivity, nitrogen balance and economics of winter maize (Zea mays) as influenced by QPM cultivars and nitrogen levels. Indian Journal of Agricultural Sciences. 84(2): 306-308.

Paolo, E.D and Rinaldi, M. 2008. Yield response of corn to irrigation and nitrogen fertilization in a Mediterranean environment. Field Crops Research. 105: 202-210.

Paramasivan, M., Malarvizhi, $\mathrm{P}$ and Tiyageswari, S. 2013. Effect of balanced nutrition on yield, nutrient uptake and soil fertility of maize (Zea mays L.) in an inceptisol of Tamil Nadu. The Andhra Agricultural Journal. 60(1): 64-69.

Pennaiah, H. 2005. Effect of irrigation and nutrient levels on yield and quality of rabi maize (Zea mays L.). M. Sc. Thesis submitted to Acharya N.G. Ranga Agricultural University, Rajendranagar, Hyderabad, Andhra Pradesh. 
Reddy, M.M and Padmaja, B. 2014. Productivity and water use efficiency of no-till winter (rabi) maize (Zea mays) as influenced by drip-fertigation. Indian Journal of Agronomy. 59(1): 96-100.

Reddy, M.M., Padmaja, B and Reddy, D.V.V. 2012. Response of maize to irrigation scheduling and nitrogen doses under no-till condition in rice fallows. Journal of Research ANGRAU. 40(1): 6-12.

Sharma, A.R and Behera, U.K.2009. Nitrogen contribution through Sesbania green manure and dual-purpose legumes in maize-wheat cropping system: agronomic and economic considerations. Plant and Soil. 325: 289-304.
Singh, S.D. 2001. Effect of different irrigation regimes and nitrogen levels on growth, yield and quality of baby corn. Madras Agricultural Journal. 88(7-9): 367370.

Viswanatha, G.B., Ramachandrappa, B.K and Nanjappa, H.V. 2002. Soil-plant water status and yield of sweet corn (Zea mays L. cv. Saccharata) as influenced by drip irrigation and planting methods. Agricultural Water Management. 55:85-91.

Zakkam, M., Chandrasekhar, K and Subbaiah, G. 2012. Response of maize (Zea mays L.) to planting densities and nitrogen levels under late rabi conditions. The Andhra Agricultural Journal. 59(4): 517-519.

\section{How to cite this article:}

Deepthi Kiran, Y., V. Sumathi and Prabhakara Reddy, G. 2019. Yield and Economics of Maize (Zea mays) Preceded by Greengram as Influenced by Irrigation Schedules and Nitrogen Levels. Int.J.Curr.Microbiol.App.Sci. 8(11): 556-567. doi: https://doi.org/10.20546/ijcmas.2019.811.068 